PROCEEDINGS OF THE

AMERICAN MATHEMATICAL SOCIETY

Volume 139, Number 4, April 2011, Pages 1273-1276

S 0002-9939(2010)10662-7

Article electronically published on September 30, 2010

\title{
GAUSS SUMS OVER FINITE FIELDS AND ROOTS OF UNITY
}

\author{
ROBERT J. LEMKE OLIVER
}

(Communicated by Matthew A. Papanikolas)

\begin{abstract}
Let $\chi$ be a non-trivial character of $\mathbb{F}_{q}^{\times}$, and let $g(\chi)$ be its associated Gauss sum. It is well known that $g(\chi)=\varepsilon(\chi) \sqrt{q}$, where $|\varepsilon(\chi)|=1$. Using the $p$-adic gamma function, we give a new proof of a result of Evans which gives necessary and sufficient conditions for $\varepsilon(\chi)$ to be a root of unity.
\end{abstract}

\section{IntRoduction AND STATEMENT OF RESUlts}

Let $p>2$ be a prime, and let $q=p^{f}$ for some $f \geq 1$. Let $\psi: \mathbb{F}_{p} \rightarrow \mathbb{C}^{\times}$be a non-trivial additive character, and let $\chi: \mathbb{F}_{q}^{\times} \rightarrow \mathbb{C}^{\times}$be a non-trivial multiplicative character. The Gauss sum $g(\chi)=g(\chi, \psi)$ associated to $\chi$ is given by

$$
g(\chi):=\sum_{x \in \mathbb{F}_{q}^{\times}} \chi(x) \psi(\operatorname{tr}(x)),
$$

where $\operatorname{tr}(x):=x+x^{p}+\ldots+x^{p^{f-1}}$. The determination of $g(\chi)$ is of central importance in analytic number theory, as it reflects both the multiplicative and additive structure of $\mathbb{F}_{q}$. Classical arguments show that $|g(\chi)|=\sqrt{q}$. On the other hand, the quantity $\varepsilon(\chi):=g(\chi) / \sqrt{q}$ has only been determined for $\chi$ of certain orders (see [1 for a comprehensive treatment of recent results). Motivated by private communications with Zagier, we determine when $\varepsilon(\chi)$ is a root of unity.

Theorem 1.1. Let $\chi: \mathbb{F}_{q}^{\times} \rightarrow \mathbb{C}^{\times}$be a multiplicative character of order $m$ and let $r$ be the order of $p$ modulo $m$. The quantity $\varepsilon(\chi)$ is a root of unity if and only if for every integer $t$ coprime to $m$ we have that

$$
\sum_{i=0}^{r-1} \overline{t p^{i}}=\frac{r m}{2}
$$

where $\overline{t p^{i}}$ denotes the canonical representative of $t p^{i}$ modulo $m$ in $[0, \ldots, m-1]$.

Remark. After this work was done, the author learned that Theorem 1.1 was first obtained by Evans [2]. Evans's proof used Stickelberger's relation on the decomposition of $g(\chi)$ into prime ideals (see [4]). An equivalent condition, essentially (2.5) below, was later obtained by Yang and Zheng [5, again using Stickelberger's relation. We give a different proof of Theorem 1.1, one based on a deep theorem of Gross and Koblitz [3] relating Gauss sums to the $p$-adic gamma function.

Received by the editors April 22, 2010.

2010 Mathematics Subject Classification. Primary 11T24.

Key words and phrases. Gauss sums, Gross-Koblitz.

(C)2010 American Mathematical Society 


\section{Proof of Theorem 1.1}

In Section 2.1 we begin by defining the $p$-adic gamma function $\Gamma_{p}(z)$. We then state the Gross-Koblitz formula, which relates Gauss sums over a finite field to a product of values of $\Gamma_{p}(z)$. In Section 2.2 we apply the Gross-Koblitz formula to prove Theorem 1.1 .

2.1. The Gross-Koblitz formula. Let $p>2$ be a prime and $q=p^{f}$ for some $f \geq 1$. The $p$-adic gamma function $\Gamma_{p}(z): \mathbb{Z}_{p} \rightarrow \mathbb{Z}_{p}^{\times}$is defined by

$$
\Gamma_{p}(z):=\lim _{\substack{m \rightarrow z \\ m \in \mathbb{Z}}}(-1)^{m} \prod_{\substack{j<m \\(j, p)=1}} j .
$$

Let $\omega_{f}: \mathbb{F}_{q}^{\times} \rightarrow \mathbb{C}^{\times}$be the Teichmüller character of $\mathbb{F}_{q}, \psi: \mathbb{F}_{p} \rightarrow \mathbb{C}^{\times}$be a nontrivial additive character, and $\zeta_{p}=\psi(1)$. Let $\pi \in \mathbb{Q}_{p}\left(\zeta_{p}\right)$ be the unique element satisfying both $\pi^{p-1}=-p$ and $\zeta_{p} \equiv 1+\pi\left(\bmod \pi^{2}\right)$. For integers $0 \leq a<q-1$, the Gauss sum $g\left(\omega_{f}^{-a}\right)$ is defined by

$$
g\left(\omega_{f}^{-a}\right):=-\sum_{x \in \mathbb{F}_{q}^{\times}} \omega_{f}^{-a}(x) \psi(\operatorname{tr}(x)),
$$

where $\operatorname{tr}(x):=x+x^{p}+\ldots+x^{p^{f-1}}$. The Gross-Koblitz formula 3] states that

$$
g\left(\omega_{f}^{-a}\right)=\pi^{S(a)} \prod_{j=0}^{f-1} \Gamma_{p}\left(\left\{\frac{a p^{j}}{q-1}\right\}\right)
$$

where $S(a)$ denotes the sum of digits in the base $p$ expansion of $a$ and, for any $x \in \mathbb{R},\{x\}:=x-\lfloor x\rfloor$ denotes the fractional part of $x$.

2.2. Proof of Theorem 1.1. Let $\chi$ be a multiplicative character of $\mathbb{F}_{q}^{\times}$of order $m$. There is a unique $a$ such that $0 \leq a<q-1$ and $\chi=\omega_{f}^{-a}$. Since $g(\chi) \in \mathbb{Q}\left(\zeta_{p}, \zeta_{q-1}\right)$, $\varepsilon(\chi)$ is a root of unity if and only if $g(\chi)^{2 p(q-1)}=q^{p(q-1)}$. The Gross-Koblitz formula (2.3) yields that

$$
g(\chi)^{2 p(q-1)}=p^{2 p(q-1) S(a) /(p-1)}\left(\prod_{j=0}^{f-1} \Gamma_{p}\left(\left\{\frac{a p^{j}}{q-1}\right\}\right)\right)^{2 p(q-1)}
$$

and by comparing the $p$-adic valuation of both sides, we see that a necessary condition for $\varepsilon(\chi)$ to be a root of unity is $S(a)=\frac{f(p-1)}{2}$. In fact, if $\chi^{\prime}$ is another character of $\mathbb{F}_{q}^{\times}$of order $m$, then there is an element of $\operatorname{Gal}\left(\mathbb{Q}\left(\zeta_{p}, \zeta_{m}\right)\right)$ taking $g(\chi)$ to $g\left(\chi^{\prime}\right)$. Hence, $\varepsilon(\chi)$ is a root of unity if and only if $\varepsilon\left(\chi^{\prime}\right)$ is as well. Thus, if $\varepsilon(\chi)$ is a root of unity, for all $t$ coprime to $m$ we have that

$$
S\left(\overline{t a}^{(q-1)}\right)=\frac{f(p-1)}{2},
$$

where $\overline{t a}^{(q-1)}$ is the canonical reduction of $t a$ modulo $q-1$. This condition will prove to be sufficient to guarantee that $\varepsilon(\chi)$ is a root of unity. To see this, we begin by reinterpreting the sum of digits function $S(a)$. 
Lemma 2.1. For any $0 \leq b<q-1$, we have that

$$
\sum_{j=0}^{f-1}\left\{\frac{b p^{j}}{q-1}\right\}=\frac{S(b)}{p-1} .
$$

Proof. Write $b=\sum_{i=0}^{f-1} b_{i} p^{i}$. For any $0 \leq j \leq f-1$, we observe that $b p^{j} \equiv b^{(j)}$ $(\bmod q-1)$, where $0 \leq b^{(j)}<q-1$ is the $j$-th iterate of the cyclic permutation on the base $p$ digits of $b$. Hence, we have that

$$
\begin{aligned}
\sum_{j=0}^{f-1}\left\{\frac{b p^{j}}{q-1}\right\} & =\frac{1}{q-1} \sum_{j=0}^{f-1} b^{(j)} \\
& =\frac{S(b)}{p-1} .
\end{aligned}
$$

Write $a=t_{0} \cdot(a, q-1)$ for some $t_{0}$ coprime to $m$. Since $m=\frac{q-1}{(a, q-1)}$, we have that

$$
\left\{\frac{a p^{j}}{q-1}\right\}=\left\{\frac{t_{0} p^{j}}{m}\right\}=\frac{\overline{t_{0} p^{j}}}{m}
$$

whence

$$
\sum_{j=0}^{f-1}\left\{\frac{a p^{j}}{q-1}\right\}=\frac{f}{r} \sum_{j=0}^{r-1} \frac{\overline{t_{0} p^{j}}}{m}
$$

where $\overline{t p^{j}}$ is the reduction of $t p^{j}$ modulo $m$ and $r$ is the multiplicative order of $p$ modulo $m$. Hence, by Lemma 2.1 (2.5) holds for $t$ coprime to $m$ if and only if we have that

$$
\sum_{j=0}^{r-1} \overline{t p^{j}}=\frac{r m}{2} .
$$

This establishes the necessity of (1.2) in the statement of Theorem 1.1. Sufficiency follows immediately from a result of Gross and Koblitz [3]: If $\left\{a_{1}, \ldots, a_{k}, n_{1}, \ldots, n_{k}\right\}$ is a set of integers such that, for all $u$ coprime to $m, \sum_{i=1}^{k} n_{i} \cdot \overline{u a_{i}}$ is an integer independent of $u$, then the product $\prod_{i=1}^{k} \prod_{j=0}^{f-1} \Gamma_{p}\left(\frac{\overline{a_{i} p^{j}}}{m}\right)^{n_{i}}$ is a root of unity. We apply this result with $k=r, a_{i}=p^{i}$, and $n_{i}=2$, showing that if (1.2) is satisfied, then $\varepsilon(\chi)$ is a root of unity.

\section{REFERENCES}

[1] B. C. Berndt, R. J. Evans, and K. S. Williams. Gauss and Jacobi sums. Canadian Mathematical Society Series of Monographs and Advanced Texts. John Wiley \& Sons Inc., New York, 1998. A Wiley-Interscience Publication. MR.1625181 (99d:11092)

[2] R. J. Evans. Generalizations of a theorem of Chowla on Gaussian sums. Houston J. Math., 3(3):343-349, 1977. MR0498491 (58:16600)

[3] B. H. Gross and N. Koblitz. Gauss sums and the p-adic $\Gamma$-function. Ann. of Math. (2), 109(3):569-581, 1979. MR534763 (80g:12015) 
[4] K. Ireland and M. Rosen. A classical introduction to modern number theory, volume 84 of Graduate Texts in Mathematics. Springer-Verlag, New York, second edition, 1990. MR.1070716 (92e:11001)

[5] J. Yang and W. Zheng. On a theorem of Chowla. J. Number Theory, 106(1):50-55, 2004. MR2049591 (2005b:11197)

Department of Mathematics, University of Wisconsin, Madison, Wisconsin 53706

Current address: Department of Mathematics and Computer Science, Emory University, Atlanta, Georgia 30322

E-mail address: lemkeoliver@gmail.com 